\title{
Medical Image of the Week: Cannon V Waves
}

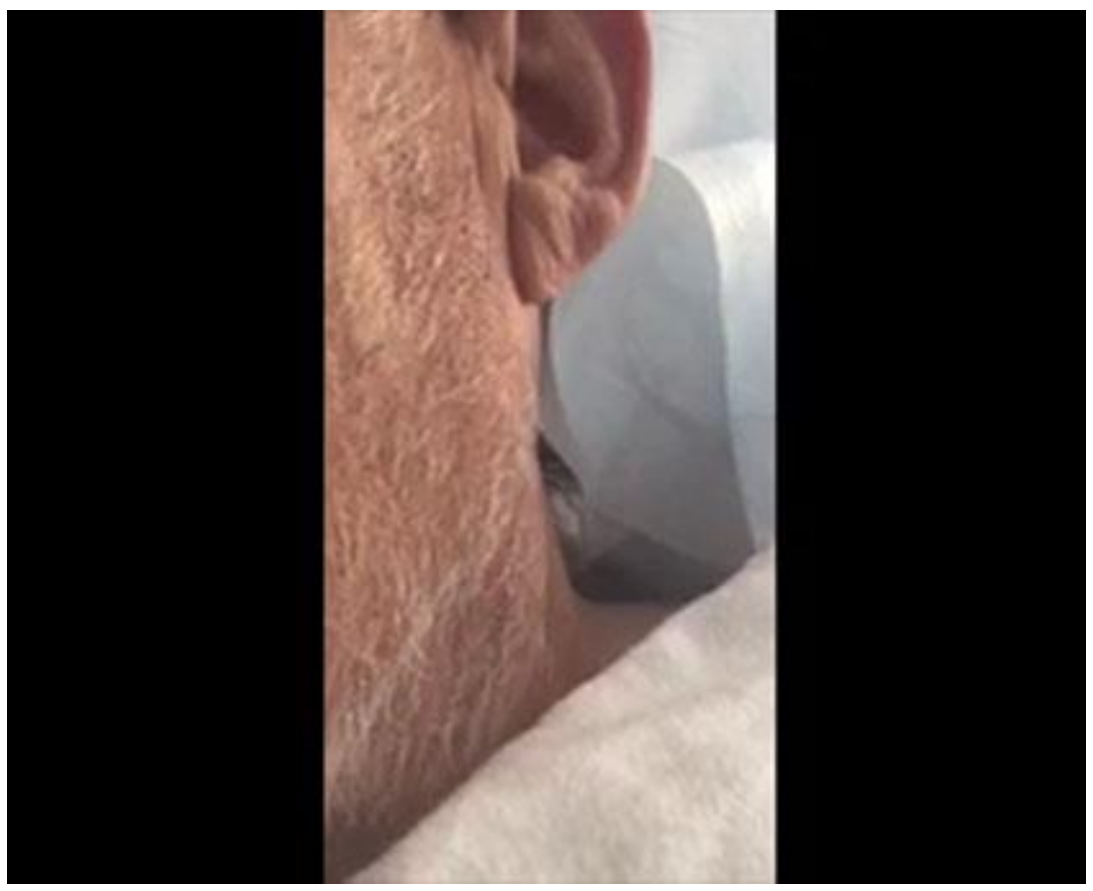

Figure 1. Static image from video showing jugular venous distention to earlobes with cannon $\mathrm{V}$ waves.

A 66-year-old man experienced recurrent ascites of unknown etiology over six months. He had previously undergone a renal transplant secondary to complications of diabetes and hypertension and had known severe coronary artery disease. His most recent paracentesis revealed an albumin $1.6 \mathrm{~g} / \mathrm{dL}$ (serum albumin 2.1) and a total protein of $3.8 \mathrm{~g} / \mathrm{dL}$. His adenosine deaminase was $11.6 \mathrm{U} / \mathrm{L}$ (normal $<7.6 \mathrm{U} / \mathrm{L}$ ), but repeated bacterial and mycobacterial ascites cultures were negative, as were a carcinoembryonic antigen assay and ascites cytology. Computerized tomography of the abdomen showed findings consistent with cirrhosis, but an extensive workup for common causes of cirrhosis was negative.

Physical exam showed jugular venous distention with prominent $\mathrm{V}$ waves and a holosystolic murmur at the left lower sternal border (Figure 1). Echocardiography showed a dilated right ventricle, moderate pulmonary and tricuspid regurgitation and an estimated right ventricular systolic pressure of $87 \mathrm{~mm} \mathrm{Hg}$. Cardiac catherization confirmed the presence of an elevated right ventricular pressure of $72 / 10(22) \mathrm{mm} \mathrm{Hg}$, an elevated pulmonary artery pressure of $75 / 27(45) \mathrm{mm} \mathrm{Hg}$ and a left ventricular ejection fraction of $20-25 \%$. The right atrial pressure was 20 and the pulmonary artery occlusion pressure was $22 \mathrm{mmHg}$. A diagnosis of pulmonary hypertension secondary to left ventricular heart disease (type 2 pulmonary hypertension) with congestive hepatopathy and cardiac ascites was made. 
The patient's physical examination provided an important clue to the etiology of the ascites - cardiac ascites is thought to be due to chronic venous congestion of the liver due to transmission of high central venous pressures. Tricuspid regurgitation can be associated with severe hepatic congestion because of retrograde transmission of right ventricular pressure directly into the hepatic veins. In some patients (although not in this patient), careful examination will reveal that the liver in such patients is palpably pulsatile.

Cardiac ascites is typically characterized by a serum albumin gradient (SAAG) $\geq 1.1 \mathrm{~g} / \mathrm{dL}$ (indicative of portal hypertension) and ascites protein level of $\geq 2.5 \mathrm{~g} / \mathrm{dL}$ (1). We cannot fully explain why this patient's SAAG was low. A complete workup for infectious and oncological etiologies of low SAAG ascites was negative. It has been noted that in patients with known cirrhosis (as in this patient), the finding of a low SAAG has a low specificity for infectious and oncological etiologies of ascites (2). Serositis which can sometimes manifest as ascites can also be a complication of tacrolimus which the patient was receiving $s / p$ renal transplant. It's possible that tacrolimus might have changed the nature of the ascites fluid in this patient but this is conjectural.

Robert A. Raschke, MD College of Medicine-Phoenix

Phoenix, AZ USA

\section{References}

1. Sam AH, James THT. Rapid Medicine. Wiley-Blackwell; 2009: ISBN 1-40518323-3.

2. Khandwalla HE, Fasakin $\mathrm{Y}$, El-Serag HB. The utility of evaluating low serum albumin gradient ascites in patients with cirrhosis. Am J Gastroenterol. 2009 Jun;104(6):1401-5. [CrossRef] [PubMed] 